\title{
piRNA dynamics in divergent zebrafish strains reveal long-lasting maternal influence on zygotic piRNA profiles
}

\author{
LUCAS J.T. KAAIJ, SUZANNE W. HOOGSTRATE, EUGENE BEREZIKOV, and RENÉ F. KETTING ${ }^{1,2}$ \\ Hubrecht Institute-KNAW and University Medical Centre Utrecht, 3584 CT Utrecht, The Netherlands
}

\begin{abstract}
Transposable elements (TEs) are mobile genetic elements that can have many deleterious effects on the fitness of their host. The germline-specific PIWI pathway guards the genome against TEs, deriving its specificity from sequence complementarity between PIWI-bound small RNAs (piRNAs) and the TEs. The piRNAs are derived from so-called piRNA clusters. Recent studies have demonstrated that the piRNA repertoire can be adjusted to accommodate recent TE invasions by capturing invading TEs in piRNA loci. Thus far, no information concerning piRNA divergence is available from vertebrates. We present piRNA analyses of two relatively divergent zebrafish strains. We find that significant differences in the piRNA populations have accumulated, most notably among active class I TEs. This divergence can be split into differences in piRNA abundance per element and differences in sense/antisense polarity ratios. In crosses between animals of the different strains, many of these differences are resolved in the progeny. However, some differences remain, often leaning to the maternally contributed piRNA population. These differences can be detected at least two generations later. Our data illustrate, for the first time, the fluidity of piRNA populations in vertebrates and how the established diversity is transmitted to future generations.
\end{abstract}

Keywords: piRNAs; transposons; hybrids; maternal effect; zebrafish

\section{INTRODUCTION}

Transposable elements (TEs) make up a large proportion of the genome of many organisms. The activation of transposons and the subsequent integration of mobile elements into new genomic loci must be tightly controlled, as this can result in the disruption of protein-coding genes and other deleterious events (Burns and Boeke 2012). TEs can be divided into two classes based on structure and mechanism of transposition. Retro-transposons (class I) transpose via an RNA intermediate. Because of this, transposition events generate additional copies at new genomic locations. Most DNA transposons (class II) are excised from the genome and integrate elsewhere in the genome, a process that does not necessarily result in a net increase in copy number (Malone and Hannon 2009; Burns and Boeke 2012). Over the course of evolution the genomes of most species have become littered with TE copies, many of which are no longer active because they carry many mutations. In most vertebrate genomes, including that of zebrafish, active copies have only been detected from the class I family of TEs, while no functional class II elements appear to be present anymore.

\footnotetext{
${ }^{1}$ Present address: Institute for Molecular Biology, D-55128 Mainz, Germany

${ }^{2}$ Corresponding author

E-mail r.ketting@hubrecht.eu

Article published online ahead of print. Article and publication date are at http://www.rnajournal.org/cgi/doi/10.1261/rna.036400.112.
}

In recent years a germline-specific small RNA-based defense mechanism against transposable elements has been identified: the PIWI pathway (Vagin et al. 2006; Aravin et al. 2007a,b; Brennecke et al. 2007; Gunawardane et al. 2007). The core proteins of this pathway are members of the Piwi subclade of Argonaute proteins (Hammond et al. 2001; Carthew and Sontheimer 2009). These proteins directly interact with small RNA molecules that guide the Argonaute proteins sequence specifically to their target RNAs. Piwi was initially described in Drosophila, where mutants showed defects in female germline maintenance (Cox et al. 1998). This is accompanied by the desilencing of transposons (Cox et al. 1998; Sarot et al. 2004; Vagin et al. 2006; Brennecke et al. 2007). In contrast to miRNAs, the PIWI-bound small RNAs, or piRNAs, are Dicer independent (Vagin et al. 2006; Houwing et al. 2007), and many aspects of their biogenesis remain unsolved. PiRNAs are generated through endonucleolytic cleavage of long single-stranded transcripts, followed by binding of a Piwi protein to the $5^{\prime}$ end of the cleavage site and trimming of the corresponding $3^{\prime}$ end by an exonuclease (Brennecke et al. 2007; Gunawardane et al. 2007; Kawaoka et al. 2011). This process is most likely responsible for the rather wide size-range of piRNAs between 25 and 30 nucleotides (nt), and the size distribution profiles that are quite specific for individual Piwi protein family members (Aravin et al. 2006; Girard et al. 2006; Brennecke et al. 2007; Gunawardane et al. 2007; Houwing et al. 2008; Huang et al. 2011). 
This general piRNA biogenesis mechanism can be divided into two distinct variants. In the first variant, long transcripts derived from genomic clusters that are rich in transposon sequences are cleaved by an endonuclease (Brennecke et al. 2007). Zucchini is the prime candidate for carrying out this step (Ipsaro et al. 2012; Nishimasu et al. 2012; Voigt et al. 2012). The piRNAs from this mode of biogenesis display a strong bias for an Uracil at their $5^{\prime}$ end, possibly reflecting the preference of the relevant Piwi proteins for $5^{\prime} U$ containing RNA fragments (Kawaoka et al. 2011). The loci from which these transcripts are derived, also named piRNA clusters, serve as a genetic memory of transposition activity in the past, and the ability of an organism to silence transposons using the PIWI-piRNA pathway lies in the presence of complementary sequences in these piRNA clusters (Pelisson et al. 1994; Malone and Hannon 2009). These clusters appear to be marked by specific HP1-like proteins that may direct the transcripts from these loci to piRNA biogenesis factories (Klattenhoff et al. 2009; Zhang et al. 2012).

In the second variant, also named ping-pong or secondary biogenesis, the endonucleolytic cleavage is performed by a Piwi-piRNA complex. This results in a population of piRNAs with opposite strand polarity, which is characterized by a bias for Adenosine at position 10, reflecting the cleavage specificity of Argonaute proteins (Brennecke et al. 2007; Gunawardane et al. 2007). The first type of piRNAs is often referred to as primary piRNAs, the second population as secondary piRNAs. Because of their biogenesis, piRNAs from both pathways tend to overlap $10 \mathrm{nt}$ at their $5^{\prime}$ ends, and the frequency of such overlaps, also referred to as "ping-pong" signal, is often taken as a measure for Piwi pathway activity. The two types of piRNAs are usually bound by different Piwi family members.

The zebrafish genome encodes two PIWI proteins, named Ziwi and Zili, which are both expressed in the female and the male germ cells (Houwing et al. 2007, 2008). Mutations in either one of these two PIWI genes, and many PIWI associated proteins, result in sterility (Houwing et al. 2007, 2008; Kamminga et al. 2010; Huang et al. 2011). Ziwi is a cytoplasmic protein and binds primary piRNAs. These Ziwi-bound piRNAs are strongly enriched to be antisense with regard to TE mRNAs (Houwing et al. 2007, 2008). The zygote receives both Ziwi and Ziwi-bound piRNAs via the mother, and it has been suggested that these may protect the zygote against transposition events during early development (Houwing et al. 2007, 2008). Zili is only expressed zygotically, can be both cytoplasmic and nuclear, and is loaded with secondary sense piRNAs (Houwing et al. 2007, 2008).

In order to accommodate the constantly evolving sequence landscape of endogenous and newly invading TEs, the sequence contents of piRNA clusters would be expected to be rather flexible. Indeed, such flexibility has been documented in recent studies. First, attempts to generate transgenic silkworm cell lines showed a remarkable incidence of transgenes integrating into piRNA clusters, supporting the idea that piRNA clusters act as a sink for active transposable elements
(Kawaoka et al. 2012). Furthermore, studies in mouse and Drosophila showed that inserting GFP sequences in piRNA clusters resulted in GFP-derived piRNAs and stable silencing of GFP in trans (Muerdter et al. 2012). Finally, the recent invasion of P-elements that has occurred in wild populations of Drosophila after most laboratory strains had been isolated has allowed direct demonstration of piRNA cluster adaptation in response to the invasion of a novel TE. This invasion resulted not only in integration of active copies into the genome, but also in the incorporation of P-element sequences in piRNA clusters, followed by P-element-specific piRNA production and P-element silencing (Pelisson 1981; Kidwell 1983; Bucheton et al. 1984; Sang et al. 1984; Brennecke et al. 2008; Khurana et al. 2011).

So far, most studies investigating natural piRNA divergence, maternal inheritance, and transposon silencing have been focused on Drosophila (Brennecke et al. 2008; Chambeyron et al. 2008; Rozhkov et al. 2010; Khurana et al. 2011). Questions concerning natural piRNA divergence and inheritance in vertebrate systems have not been addressed thus far. This study was designed to gain insight into the natural divergence of piRNA populations between zebrafish strains and how these differences behave in ensuing generations following crosses between such strains. We focused on two zebrafish strains, SJD and Tu, based on previous work that described high SNP frequencies between these strains (Guryev et al. 2006). We show that in zebrafish piRNA populations diverge over time and that the most dramatic differences accumulate in piRNAs derived from class I elements. Although most differences in piRNA populations are resolved in the progeny of crosses between divergent strains, detailed analysis revealed a maternal role in shaping the zygotic piRNA population. Strikingly, this maternal effect is still evident two generations later, indicating long-lasting maternal influence on zygotic piRNA populations.

\section{RESULTS}

\section{SJD and Tu strains have active piRNA pathways}

We generated small RNA sequencing data from male and female gonads isolated from SJD and Tu animals and obtained at least 16 million mappable reads per library. Since zebrafish strains have to be maintained outbred, we chose to sequence a pool of five individual males and females. These data reconfirm that the majority of repeat-derived small RNAs in zebrafish germ cells are piRNAs, since only a minor peak around 20-23 nt can be detected among reads derived from TEs (Fig. 1A). It also nicely reproduces previously described piRNA characteristics related to piRNA size, ping-pong signal, and $5^{\prime}$ nucleotide bias (Fig. 1A-C), illustrating that both strains have comparably active piRNA pathways.

As in previous studies (Houwing et al. 2008; Huang et al. 2011), ovary-derived piRNAs display a very strong bias for antisense polarity, particularly among class I TEs. This trend 


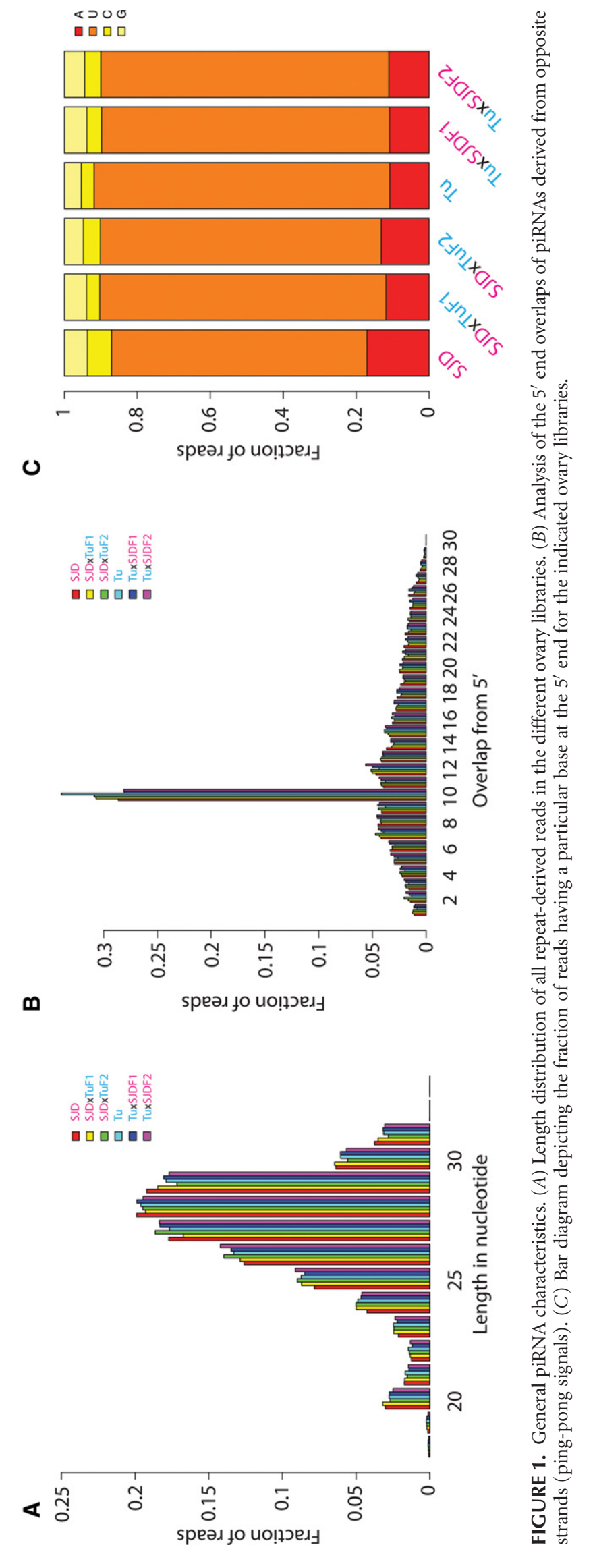


A

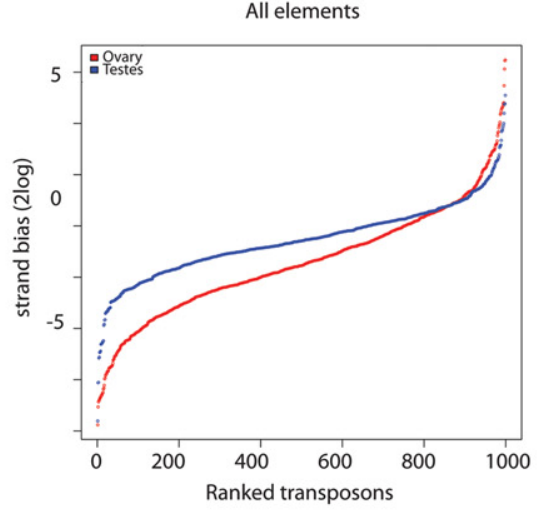

B

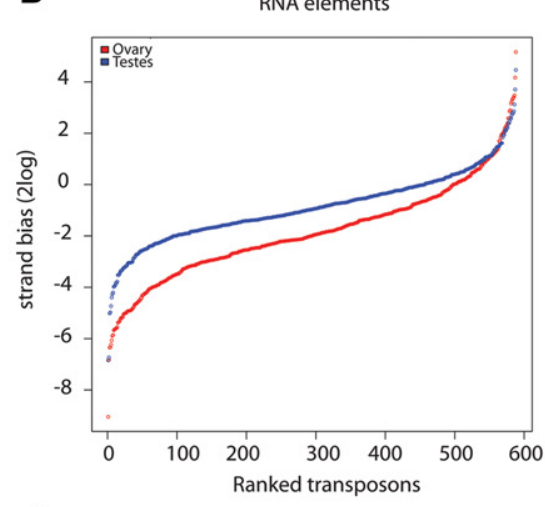

Ovary

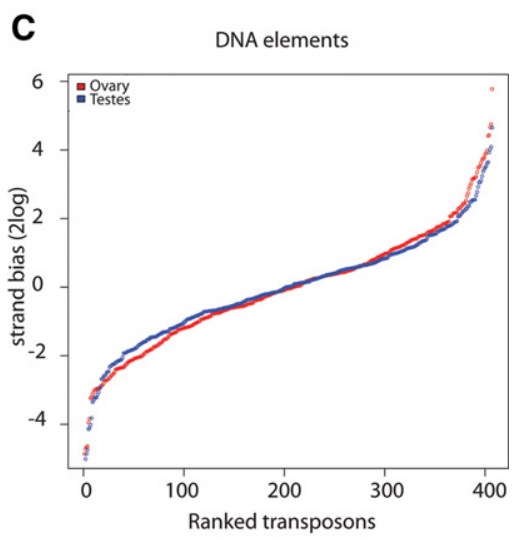

D

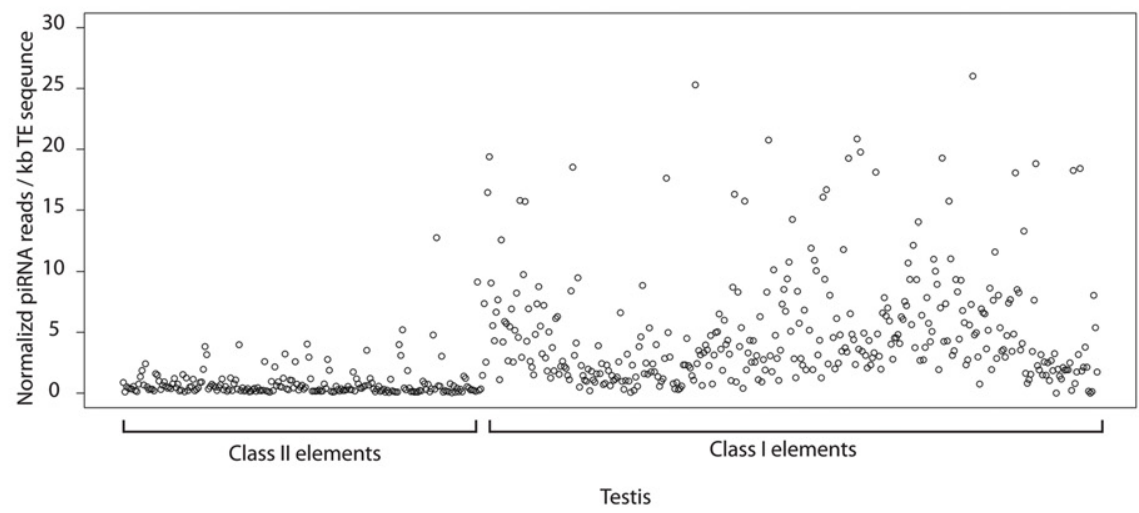

E

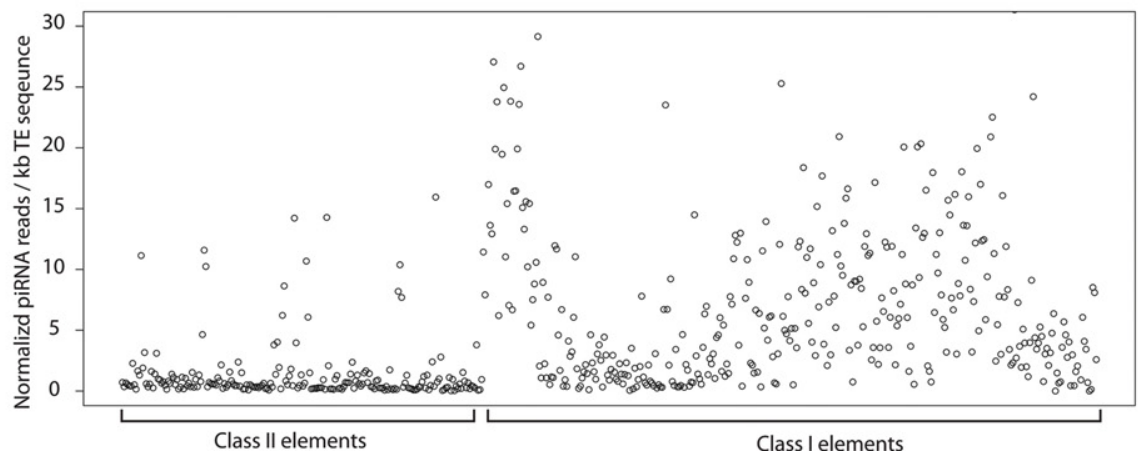

FIGURE 2. Class I and class II piRNAs. ( $A$ ) The sense/antisense strand ratios of all TEs are plotted, ranked according to increasing ratio. ( $B, C$ ) Same as $A$, but now split with regard to class I and class II TEs, respectively. $(D, E)$ piRNA abundance per TE normalized to the total amount of sequence per element in the genome in ovary $(D)$ and testis $(E)$. Every dot indicates an individual element. The plot is subdivided in two groups; RNA (class II) and DNA (class I) elements. For both the Tu and the SJD strain, similar patterns were observed. Here, only the characteristics for the Tu strain are shown.

is also detectable in testis, although not as strong (Fig. 2A-C). Class I type elements do not only display a stronger strand bias than class II elements, class I elements also produce many more piRNAs per kilobase of TE sequence present in the genome (Fig. 2D,E). All of these data are in agreement with the idea that the piRNA pathway builds up a particularly strong response against actively transposing elements.

\section{Differences between SJD and Tu piRNA populations}

To see whether any specific repeats show differential piRNA counts between the two founder strains, reads per element for both strains were normalized to the total piRNA pool and expressed as reads per million (RPM). We then displayed the normalized read count found in ovary and testis in scatter plots (Fig. 3A [ovary] and B [testis]). Most TEs have comparable read counts between the strains, and there is overall good correlation between the two piRNA populations $(R=$ 0.91 [Ovary] and $R=0.91$ [Testis]). Although piRNA abundance per element is rather similar between the two strains, some elements show big differences. To exclude artifacts arising from limited read counts, we only included TEs with at least 400 weighted reads. Between the ovaries of both strains, 32 elements show threefold differences in piRNA abundance 


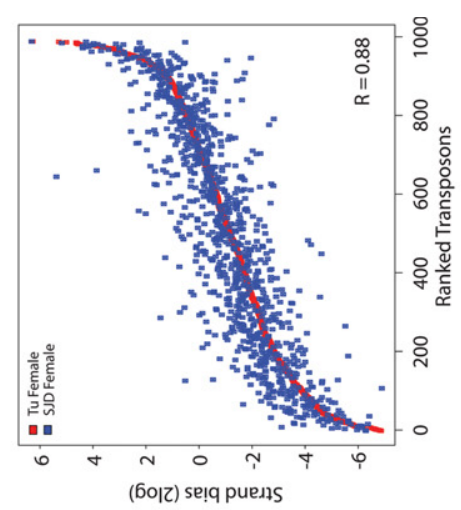

ᄂ

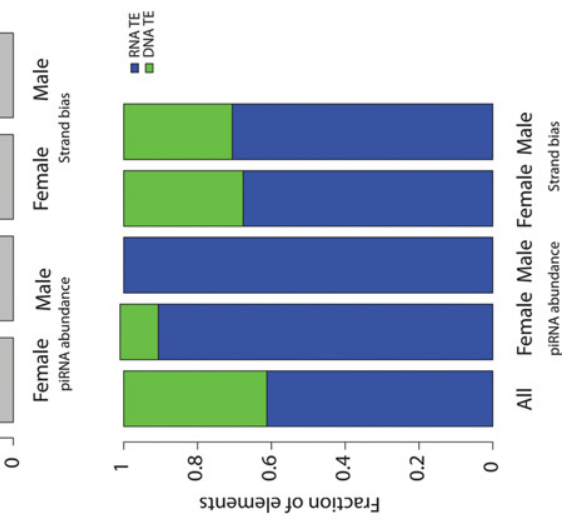

0
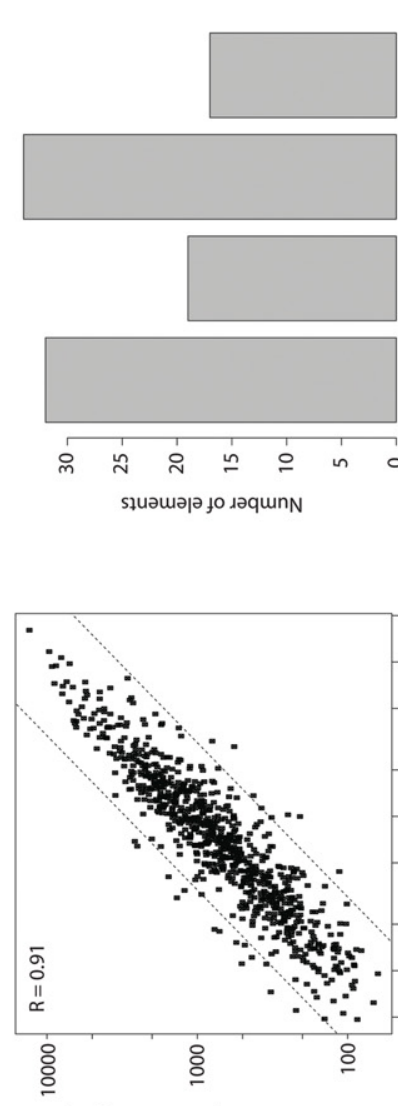

m

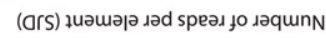

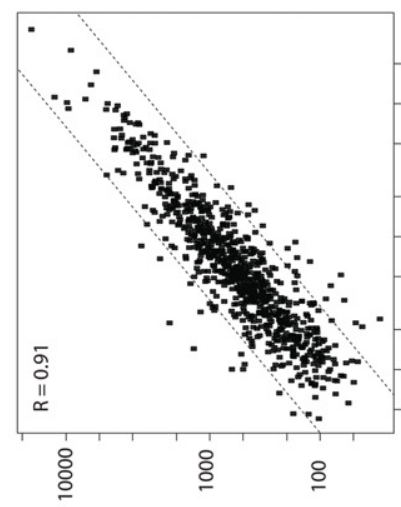

$\varangle$

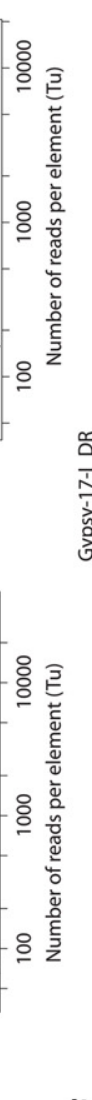

ш

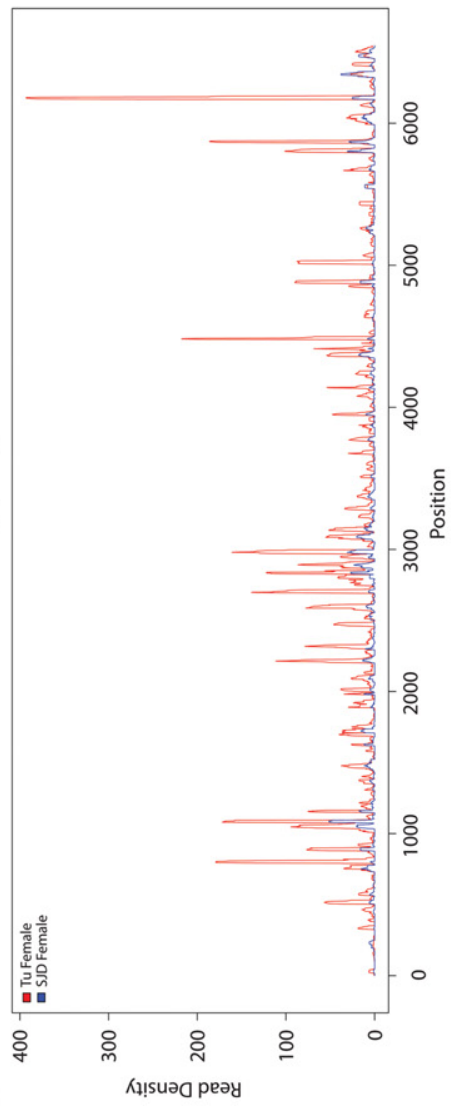

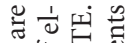

范 :

宅

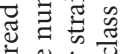

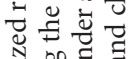

ㄱ. 잌

离至

可

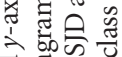

$\widetilde{\exists}: \Xi$

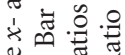

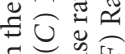

$\dot{\bar{d}}$

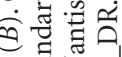

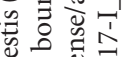

氙苞

స苞芯

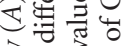

둥 000

$\exists \stackrel{\nabla}{0}$

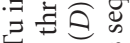

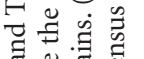

苞苛

จี $\Xi$ '्ठ

ॐै

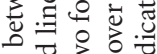

넙로엄

敢 卷

ڤั

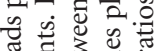

ర己

뭉

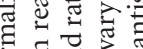

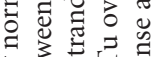

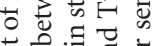

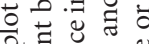

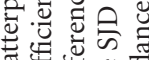

过氜

ชิ

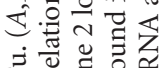

踏苛号

둥

जี \&

르릃

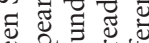

की के

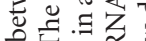

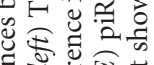

记式苞

ثี

눙요

䓀芯范

ㄴ.

м

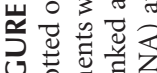

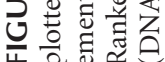


and 19 elements do so between testis populations (Fig. 3C; Supplemental Table S1). Beside differences in abundance per element, piRNA populations might also show changes in their sense/antisense ratios. Again, the correlation of these strand ratios between two strains is good, but not perfect (Fig. 3D). Thirty-four elements display a strand bias change of at least one $2 \log$ in ovary, while 17 show this in testis (Fig. 3C; Supplemental Table S2).

To rule out the possibility that the observed differences are due to technical artifacts, we generated additional libraries of the ovaries of two Tu and two SJD individuals, which were also represented in the pooled libraries. If we look specifically at the elements displaying differences in either strand ratio or piRNA abundance, we find similar effects in the libraries of all individuals as in the pooled libraries (Supplemental Fig. 1A-C). For example, of the 32 elements showing a threefold difference in piRNA abundance in the ovaries of the P0s, 30 show a twofold or more bias in the same direction in our replicas (Supplemental Fig. 1C). This attests to the reproducibility of our data and shows that the complexity of our samples is sufficient. Furthermore, if we lower the cutoff to call differentially targeted TEs to twofold, we find a dramatically lower reproducibility with 54 out of 164 elements not showing the same twofold bias or more in the individual replicas. These results indicate that a threefold cutoff for calling significant difference yields reliable results. (Supplemental Fig. 1D).

Finally, to exclude the possibility that piRNA abundance differences are simply caused by SNPs preventing proper mapping of our reads to the reference genome, we plotted the piRNA abundance observed in both founder strains over the total length of individual transposable elements. We reasoned that if the observed piRNA abundance differences relate to TE biology, we should see an effect on all piRNAs mapping to that particular TE. In contrast, if it were to be due to SNPs that prevent proper mapping, we would expect the appearance or disappearance of one major piRNA peak, while the other piRNA peaks remain unaffected. For the elements showing threefold difference between ovaries in the $\mathrm{P} 0$, we find that 25 out of 32 show consistent piRNA abundance differences over the entire length of the element, strongly suggesting that the observed piRNA abundance differences are not due to SNPs. In seven out of 32 we cannot safely exclude the existence of SNPs that might result in the observed effect, as there are only a limited number of piRNA peaks in the element (Fig. 3E; Supplemental Fig. 2). For the elements that show a threefold difference between testes in the parent strains, we find similar numbers (Supplemental Fig. 2).

\section{piRNA abundance and strand-ratio differences affect different TEs types}

When we classify transposons by class (class I or class II), we detect a significant over-representation of class I TEs among the elements with differences in piRNA abundance (Fig. 3F).
For instance, while the fraction of class I TEs among all TEs analyzed is $0.61,29$ of the 32 elements that show a threefold difference in the ovary are class I elements, and all (19 out of 19) TEs that show this in testis are class I elements $(P<$ 0.0002 and $P<0.00001$, respectively, hypergeometric distribution). Interestingly, this enrichment for class I elements is not observed among elements that change in their sense/ antisense ratio in ovary and testes $(P=0.1$ and $P=0.15$, respectively, hypergeometric distribution) (Fig. 3F). This suggests that the origins of changes in piRNA abundance and changes in strand ratios are distinct. The enrichment for class I TEs among TEs displaying differences in piRNA abundance is in line with the idea that only class I elements are active in the zebrafish genome, allowing potential new insertions of class I TEs into differentially expressed loci. However, the lack of class I enrichment among elements that display differential strand ratios suggests that de novo transposition events cannot entirely explain the observed dynamics in piRNA populations.

\section{Ovary and testis piRNAs populations behave similarly}

If we take a closer look at the elements displaying at least a threefold difference between the strains in ovary and testes, an overlap of five elements is observed (Supplemental Fig. 3C). Although this overlap is not extremely high, this is more than expected by chance (see Supplemental text). As testis and ovary piRNA population are rather dissimilar per se, we decided to lower our stringency and analyze whether elements with threefold difference in the ovary show a similar asymmetry in testis, and vice versa. This indeed shows high consistency between gonads (Supplemental Fig. 3D,E). For example, elements that have three times more reads in ovary of SJD as compared with Tu ovary show, in 12 out of 15 of the cases, more reads in SJD testis than in Tu testis. This analysis shows that the accumulated differences in piRNA abundance are similar between germ cells of the SJD and Tu strains. See Supplemental text for a more in-depth discussion of this analysis.

\section{piRNA abundance analysis in Tu/SJD cross progeny}

Given the differences in piRNA populations between the SJD and $\mathrm{Tu}$ strains, we next asked how piRNA populations behave in progeny derived from crosses between SJD and Tu. We derived such cross progeny from both reciprocal crosses. In addition to the F1 progeny, we also raised an F2 generation derived from in-crosses between F1 animals (Fig. 4A). Ovarian small RNA libraries were generated from these generations and sequenced on an Illumina genome analyzer, resulting in at least 16 million mappable reads for all different libraries. Like the SJD and Tu piRNA profiles, the piRNA profiles from these animals did not show any obvious global defects, indicating that the piRNA pathway is operating normally (Fig. 1A-C). We did not observe any signs of hybrid 
A

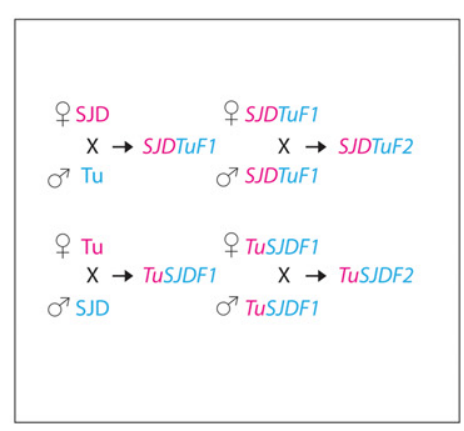

D

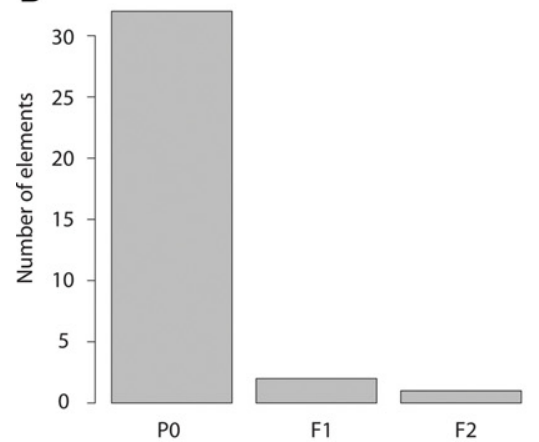

B

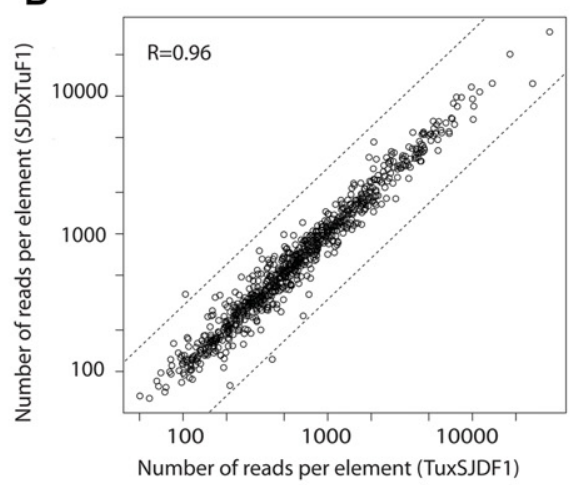

E

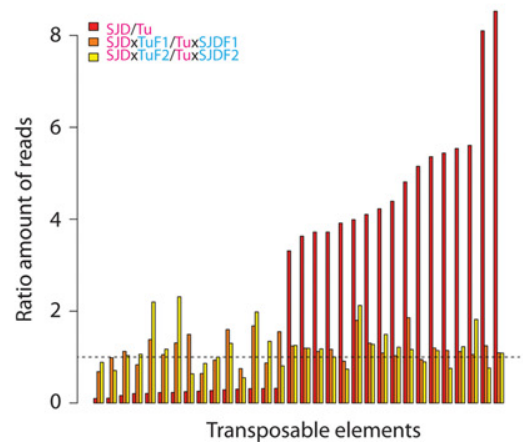

C

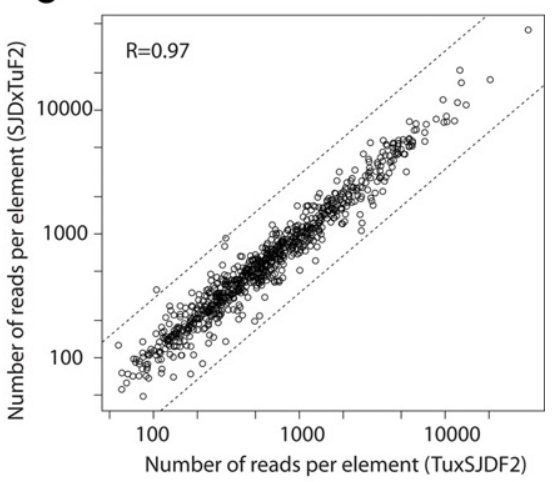

FIGURE 4. Differences in piRNA abundance between the two reciprocal crosses. (A) Scheme of the performed crosses. In italic are the crosses from which only ovarian small RNA libraries were prepared. $(B, C)$ Scatterplot of normalized reads per TE between the F1 $(B)$ and F2 $(C)$ offspring. On the $x$ - and $y$-axis, normalized read counts are plotted in log scale. (Top, left) The Spearman correlation coefficients. Dashed lines indicate the threefold difference boundary. $(D)$ Bar diagram displaying the amount of elements with threefold difference in piRNA abundance per element within the founder generation and the F1 and F2 generations. $(E)$ Bar diagram depicting the ratio of piRNA abundance detected between the two reciprocal crosses in both the F1 and F2 generations. Only those TEs are displayed that show a threefold difference in piRNA abundance between the two founder strains.

dysgenesis, as fertility was not compromised in the F1 or F2 generations. The observed sex ratio among the offspring was normal and all animals analyzed contained fully grown gonads (data not shown).

First, we analyzed piRNA abundance per element in depth. Interestingly, between the F1 generations of both reciprocal crosses we find that the correlation is much higher $(R=$ $0.96)$ than between the founders. Similarly strong correlations are found within the F2 generations $(R=0.97)$ (Fig. $4 \mathrm{~B}, \mathrm{C})$. These data strengthen the idea that the imperfect correlation observed between the founder populations is not a technical artifact, but reflects true piRNA divergence. Accordingly, we noticed a dramatic decrease of elements with a threefold difference in piRNA abundance between the two reciprocal crosses compared with the differences observed between the founders (Fig. 4D). This suggests that most accumulated differences in piRNA abundance between the SJD and $\mathrm{Tu}$ strains are established zygotically and are not significantly influenced by parental contributions.

We next looked specifically into the 32 elements showing a threefold difference between the ovaries of the founder strains. As expected, in both the F1 and the F2 the ratios of the read counts between both reciprocal crosses are in all cas- es close to 1 (Fig. 4E), indicating that parent of origin effects are small in establishing piRNA abundance.

\section{Comparison of piRNA populations between cross progeny and founders}

One may envision two ways in which the initial differences between the two founder strains can be resolved. If both parents contribute equally to the offspring, intermediate read counts would be expected, but if one parent is dominant, skewing to one parent should be observed. Such effects cannot be extracted from the data presented in Figure 4, since we looked at the ratios of the read counts between both reciprocal crosses. While this approach nicely allows comparison between both reciprocal crosses, it does not allow direct comparison between a given offspring population and the two founders. We therefore compared read abundances within each F1 and F2 population with the read counts found in the founder strains. We focused on those TEs that show a threefold over-representation in the ovaries of one of the founder strains (Supplemental Fig. 4A,B). To visualize the results, we plotted the read count within each generation (founder [P0], F1, or F2) as a fraction of the read count 
observed in the founder with the highest read count. This analysis revealed that 11 out of the 32 TEs produce intermediate piRNA counts in the F1 and F2 compared with their founders (Supplemental Fig. 4A,B), indicating roughly equal parental contribution. Furthermore, 13 out of the 32 TEs always show a strong bias to one founder line, irrespective of which reciprocal cross is examined (Supplemental Fig. 4A, $\mathrm{B})$. These cases may point at strong, dominant TE insertion loci present in only one of both founders.

\section{piRNA strand ratio effects in SJD/Tu cross progeny}

Next, we analyzed piRNA strand ratios in the ovaries of two generations following Tu/SJD crosses. To derive a general idea of how sense/antisense ratios per element behave in the crosses, we plotted the ratios found in the F1 and F2 generations and ranked all TEs according to their ratio in the F1 and $\mathrm{F} 2$ derived from female Tu animals (Fig. 5A,B). A similar pattern emerges for sense/antisense ratios as for piRNA abundance per element; namely, strand ratios of individual TEs are similar between the founder strains $(R=0.88)$ (Fig. 3D), but virtually identical between the reciprocal F1 and F2 generations ( $R=0.96$ and $R=0.97$, respectively) (Fig. 5A,B). Also, the number of elements with one 2 log difference in strand ratios between strains decreases, going from the founder strains to the two successive generations (Fig. 5C). Although most differences in strand bias are resolved when the maternal and paternal genomes merge, some elements maintain the strand ratio that was observed in one of the parents. When we looked into three examples, we found that in all three cases the strand biases resembled the strand bias found in the maternal strain (Fig. 5D). When we represent the observed strand biases of the founder strains and the various F1 and F2 generations in a heat map representing all TEs with a strand ratio difference of at least one $2 \log$ in the founder strains, we observe an overall trend in which the maternal strand bias has a dominant influence on the strand bias observed in both the F1 and F2 generation offspring (Fig. 5E). When we present the same data in a heatmap and exclude the $\mathrm{P} 0$, the maternal influence on strand bias in the F1 and F2 becomes more apparent (Supplemental Fig. 5A,B). Furthermore, hierarchical clustering shows that the majority of the $\mathrm{F} 1$ and $\mathrm{F} 2$ piRNA populations cluster together based on the piRNA population inherited via the mother (Fig. 5F). Note that this holds true for both the sequenced piRNA pools as well as for the sequenced piRNAs from individuals. Lastly, the strand ratio found in the F1 and F2 offspring correlates better with the strand ratio found in the maternal P0 strain that they are derived from than the one used in the reciprocal cross (Supplemental Fig. 5C).

\section{DISCUSSION}

The Piwi pathway is geared to respond to changes in TE content in the genome. Hence, divergence of piRNA populations between distant strains of animals is to be expected. This has been shown most clearly in Drosophila, where the appearance of novel elements in specific strains is accompanied by the formation of novel piRNA species that are in turn required to silence the corresponding TEs. Interestingly, the piRNAs have been shown to be maternally deposited into the embryos in order to help the new generation build up a response to transposable elements. However, the Drosophila Piwi pathways are quite distinct from those observed in vertebrates. For example, while both Piwi proteins participating in the ping-pong mechanism in Drosophila are cytoplasmic, in vertebrates one of the two is nuclear. Hence, findings relating to piRNA divergence and inheritance in Drosophila may not be directly applicable to vertebrates.

Thus far these aspects of the Piwi pathway have not been studied in any vertebrate system, and it is thus unclear to what extent maternal transmission of piRNAs has an effect on the piRNA profiles of the progeny. This study was designed to shed light on the natural divergence in piRNA populations in a vertebrate and to see how such divergence evolves in following generations. To achieve this we characterized in detail the piRNA populations of two diverged zebrafish strains, SJD and $\mathrm{Tu}$, and analyzed how piRNA population differences behaved in the cross progeny from such strains. In all strains, similar piRNA size and secondary piRNA biogenesis was observed as well as similar enrichments for piRNAs derived from class I TEs, indicating that all strains are Piwi pathway proficient and that no gross effects such as hybrid dysgenesis occur (Pelisson 1981; Kidwell 1983; Bucheton et al. 1984; Sang et al. 1984; Brennecke et al. 2008; Khurana et al. 2011). However, TE-specific differences were clearly detectable. We will discuss the various findings below in more detail.

\section{Variations in piRNA abundance}

In order to match the activity of TEs, it is expected that piRNA populations continuously evolve to arm the genome against transposons at each point in time. In line with the idea that piRNA populations respond to de novo transposition events, we find that virtually all TEs that display variations in piRNA abundance are class I TEs. Class II TEs are considered to be inactive in most vertebrates, including zebrafish, and thus no transposition-mediated changes in piRNA abundance would be expected. However, at present it is still unclear how piRNA abundance can change in response to transposition. These changes may relate to novel insertions into loci that allow stronger expression, triggering more RNA production for the corresponding TE and a concomitant higher piRNA production. Additionally, novel insertions into piRNA clusters may also lead to higher piRNA production levels. In order to demonstrate such hypothesized changes, the insertion patterns and the epigenetic marks of TEs in the different strains would need to be identified, including insertions in heterochromatic regions that are most likely not, or poorly represented in the current zebrafish genome builds. 

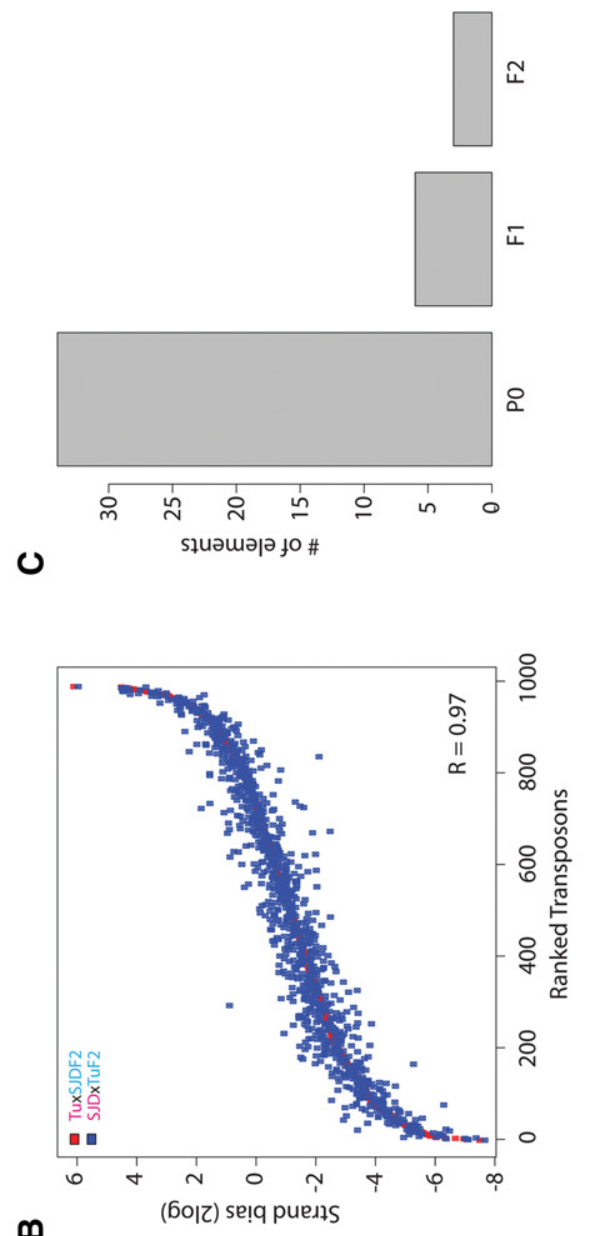

m

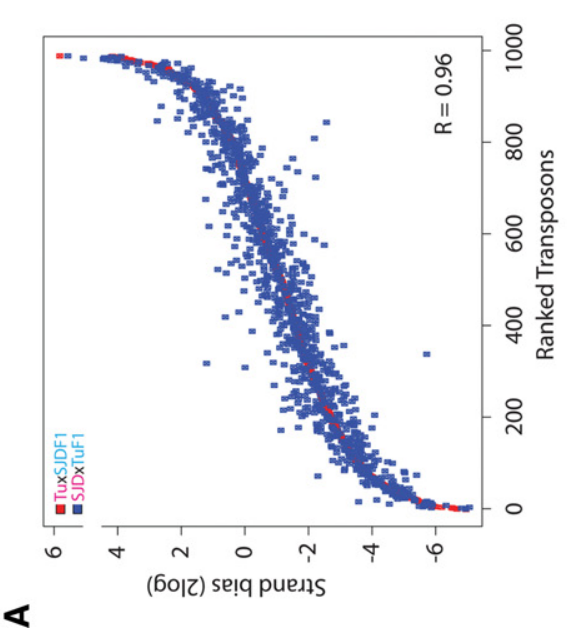

ш
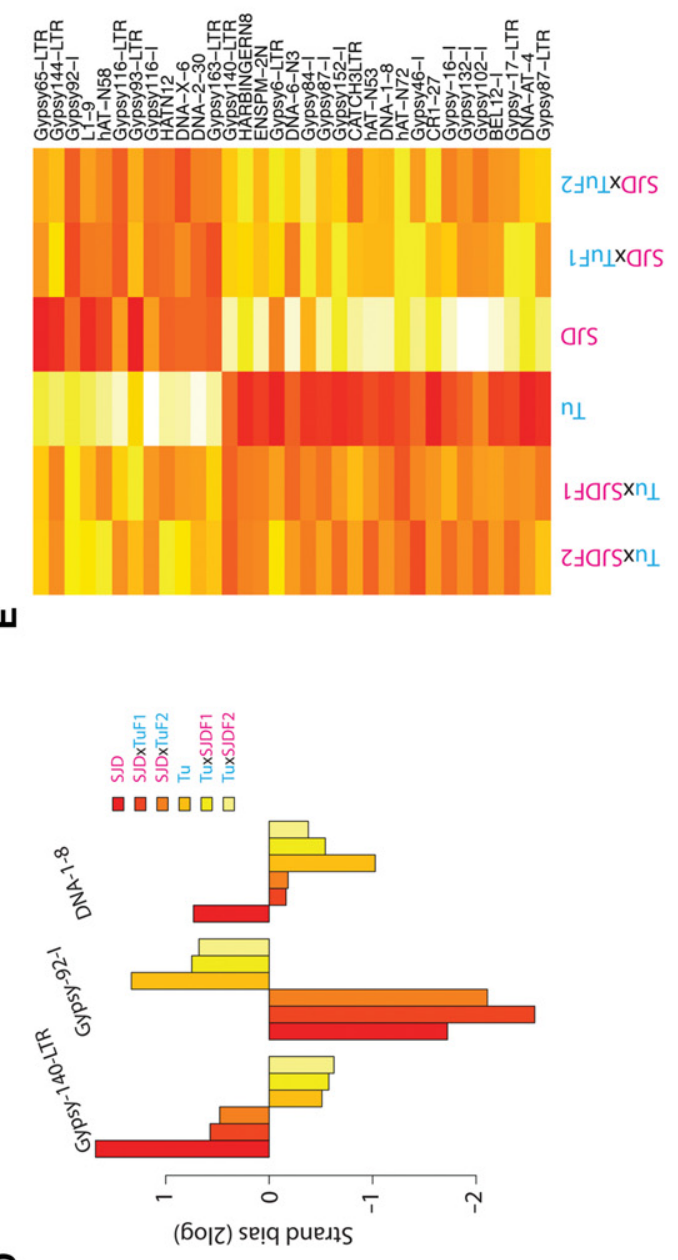

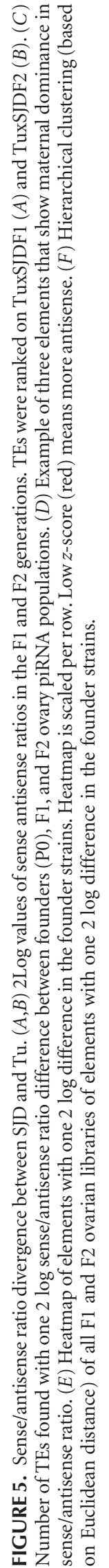




\section{Variations in piRNA strand ratios}

Strikingly, in most cases piRNA abundance differences were not accompanied by changes in strand ratio and vice versa (Supplemental Table S3). This implies that both Ziwi- and Zili-bound piRNA populations respond similarly in case piRNA abundance changes, and that strand ratio changes can occur without strong effects on piRNA abundance. Interestingly, the TEs displaying changes in strand ratios, e.g., the TEs of which the relative amounts of Ziwi- or Zili-bound piRNAs change, do not display enrichment for class I elements. This finding suggests that strand ratio changes are not directly related to novel transposition events. Rather, these changes may be related to changes in piRNA cluster behavior. These clusters, although uncharacterized in the fish, contain fragments of all transposons found in the genome, including those no longer active (Brennecke et al. 2007). Any change to a piRNA cluster affecting one strand more than the other could result in the observed effect. Alternatively, perhaps strand ratio changes reflect bystander effects in which novel insertions of class I elements lead to the funneling of sequences flanking the newly inserted copy into the piRNA pathway. Again, only an analysis of all TE insertion sites in the genomes of both founder strains would be able to shed light on the origin of strand ratio changes.

\section{Maternal effects}

Although most differences in piRNA populations that are observed between the two founder strains decrease, or disappear completely upon crossing, some parent-of-origin effects are clearly detectable. Interestingly, these do not relate to piRNA abundance, but to the ratios between sense- and antisense piRNAs. If we look into these differences in more detail, we noted an interesting and persistent bias in these strand ratios reflecting the piRNA population that was originally present in the mother. While maternal influence on piRNA abundance has been described before, maternal influence on piRNA strand ratios have not been observed before in other model systems. This points to an epigenetic role of maternally contributed piRNAs in establishing piRNA populations in the offspring, although inheritance of other epigenetic factors, like covalent histone and DNA modifications, may play a role as well.

How the maternal piRNA pool may influence the piRNA population in the gonads of adult offspring is currently unknown. Maternally deposited piRNAs and Ziwi proteins are present in zebrafish embryos (Houwing et al. 2007, 2008). However, the time between maternal piRNA deposition and the time of analysis is $>12 \mathrm{wk}$, and during this period the germline increases immensely in volume, going from tens of cells to a macroscopic structure in which most of the volume is germ-cell derived. This seems to rule out that the observed effects just reflect lingering maternal piRNA molecules. The fact that the same effect can be demonstrated even in the sec- ond generation further strengthens this idea. Rather, it is more likely that the maternally provided piRNAs should affect de novo piRNA biogenesis in the germ cells as they develop.

A possible scenario in which maternal piRNAs can shape newly generated piRNA pools in the zygote is that these maternal piRNAs affect the loading of the second Piwi protein, Zili, through secondary piRNA biogenesis (Houwing et al. 2008). Indeed, the maternally provided Ziwi protein, and most likely its associated piRNA pool, survives long enough to allow loading of Zili (Houwing et al. 2007), which only starts to be expressed $2 \mathrm{~d}$ after the PGCs have reached the gonad. Interestingly, at that time Zili enters the nucleus (Houwing et al. 2008), potentially allowing the maternal piRNA pool to have an effect on the epigenomic landscape of the germ cells, in turn affecting piRNA precursor production. It is of interest that the Zili-bound piRNA population is largely sense in orientation; i.e., Zili-piRNA complexes will not be able to recognize nascent mRNA transcripts from active TE copies. We propose that Zili may recognize piRNA cluster-derived antisense transcripts, and may thus play a role in setting the chromatin of piRNA clusters that may in turn affect piRNA biogenesis. In support of this idea, an HP-1-like protein named Rhino has been shown to interact with piRNA cluster loci and to be required for piRNA production in Drosophila (Klattenhoff et al. 2009). In addition, Drosophila Piwi has been shown to indeed affect chromatin structures at target loci (Sienski et al. 2012). This type of epigenetic modification may be very stable, as in C. elegans the Piwi pathway has been shown to initiate a form of epigenetic gene silencing that can be propagated for many generations (Ashe et al. 2012; Luteijn et al. 2012; Shirayama et al. 2012). Whether similar changes indeed underlie the effects we here describe awaits a better description of the roles of nuclear Piwi proteins in the various model systems, most notably in vertebrates.

\section{MATERIALS AND METHODS}

\section{Zebrafish strains}

Zebrafish were kept under standard conditions. SJD and Tu strains were obtained from the Zebrafish International Resource Centre (ZIRC).

\section{Crosses}

For the initial cross, female and males from SJD and Tu were set up as pairs and egg lays were harvested. After crossing, the sex of the individual fish was confirmed by dissection. The F1 from multiple pairs was grown to adulthood. The F2 incross was performed by mixing multiple family tanks of F1s and setting them up as a family.

\section{Dissection and RNA isolation}

Animals were sacrificed in ice water. Gonads were isolated and immediately transferred to Trizol (Invitrogen). RNA was isolated 
according to the manufacturer's instructions, with one exception: to increase small RNA yield, total RNA was precipitated o/n at -80 after the addition of $1 \mu \mathrm{L}$ of glycoblue (Ambion).

\section{Small RNA libraries and sequencing}

RNA from five individuals were mixed in equimolar ratio.

Small RNA libraries were prepared and sequenced as described (Huang et al. 2011).

\section{Bioinformatic analysis}

Initial bioinformatic analysis was performed as described (Huang et al. 2011). Mapping was done as previously using Blast with the word size setting $-\mathrm{W}=17$, which guarantees that all 18-mer or longer perfect matches are reported. All reads were weighed according to their mapping frequencies. Final analysis of piRNA dynamics was performed on the 1000 elements generating the most piRNA reads. The arbitrary criteria to call differential piRNA count per element were set to a threefold difference between piRNA populations. The cutoff to call strand switching was set to at least $0.52 \mathrm{log}$ strand bias and one 2 log strand ratio difference between piRNA populations. Transposon annotation was downloaded from Ensemble, but when individual elements were compared, elements with different subclassifications were collapsed. For example, elements with annotations "Gypsy-30-I_DR-int(class = LTR)" and "Gypsy-30I_DR(class = Gypsy)" were merged.

\section{DATA DEPOSITION}

Sequencing data have been deposited at GEO under accession number GSE41299.

\section{SUPPLEMENTAL MATERIAL}

Supplemental material is available for this article.

\section{ACKNOWLEDGMENTS}

The work described was funded by the following grants to R.F.K.: an ERC Starting Grant from the Ideas Program of the European Union Seventh Framework Program (202819) and the European Union Sixth Framework Program Integrated Project SIROCCO (LSHGCT-2006-037900).

Received September 14, 2012; accepted December 20, 2012.

\section{REFERENCES}

Aravin A, Gaidatzis D, Pfeffer S, Lagos-Quintana M, Landgraf P, Iovino $\mathrm{N}$, Morris $\mathrm{P}$, Brownstein MJ, Kuramochi-Miyagawa S, Nakano T, et al. 2006. A novel class of small RNAs bind to MILI protein in mouse testes. Nature 442: 203-207.

Aravin AA, Hannon GJ, Brennecke J. 2007a. The Piwi-piRNA pathway provides an adaptive defense in the transposon arms race. Science 318: 761-764.

Aravin AA, Sachidanandam R, Girard A, Fejes-Toth K, Hannon GJ. 2007b. Developmentally regulated piRNA clusters implicate MILI in transposon control. Science 316: 744-747.
Ashe A, Sapetschnig A, Weick EM, Mitchell J, Bagijn MP, Cording AC, Doebley AL, Goldstein LD, Lehrbach NJ, Le Pen J, et al. 2012. piRNAs can trigger a multigenerational epigenetic memory in the germline of C. elegans. Cell 150: 88-99.

Brennecke J, Aravin AA, Stark A, Dus M, Kellis M, Sachidanandam R, Hannon GJ. 2007. Discrete small RNA-generating loci as master regulators of transposon activity in Drosophila. Cell 128: 1089-1103.

Brennecke J, Malone CD, Aravin AA, Sachidanandam R, Stark A, Hannon GJ. 2008. An epigenetic role for maternally inherited piRNAs in transposon silencing. Science 322: 1387-1392.

Bucheton A, Paro R, Sang HM, Pelisson A, Finnegan DJ. 1984. The molecular basis of I-R hybrid dysgenesis in Drosophila melanogaster: Identification, cloning, and properties of the I factor. Cell 38: 153-163.

Burns KH, Boeke JD. 2012. Human transposon tectonics. Cell 149: $740-752$.

Carthew RW, Sontheimer EJ. 2009. Origins and mechanisms of miRNAs and siRNAs. Cell 136: 642-655.

Chambeyron S, Popkova A, Payen-Groschene G, Brun C, Laouini D, Pelisson A, Bucheton A. 2008. piRNA-mediated nuclear accumulation of retrotransposon transcripts in the Drosophila female germline. Proc Natl Acad Sci 105: 14964-14969.

Cox DN, Chao A, Baker J, Chang L, Qiao D, Lin H. 1998. A novel class of evolutionarily conserved genes defined by piwi are essential for stem cell self-renewal. Genes Dev 12: 3715-3727.

Girard A, Sachidanandam R, Hannon GJ, Carmell MA. 2006. A germline-specific class of small RNAs binds mammalian Piwi proteins. Nature 442: 199-202.

Gunawardane LS, Saito K, Nishida KM, Miyoshi K, Kawamura Y, Nagami T, Siomi H, Siomi MC. 2007. A slicer-mediated mechanism for repeat-associated siRNA $5^{\prime}$ end formation in Drosophila. Science 315: $1587-1590$.

Guryev V, Koudijs MJ, Berezikov E, Johnson SL, Plasterk RH, van Eeden FJ, Cuppen E. 2006. Genetic variation in the zebrafish. Genome Res 16: 491-497.

Hammond SM, Boettcher S, Caudy AA, Kobayashi R, Hannon GJ. 2001. Argonaute2, a link between genetic and biochemical analyses of RNAi. Science 293: 1146-1150.

Houwing S, Kamminga LM, Berezikov E, Cronembold D, Girard A, van den Elst H, Filippov DV, Blaser H, Raz E, Moens CB, et al. 2007. A role for Piwi and piRNAs in germ cell maintenance and transposon silencing in Zebrafish. Cell 129: 69-82.

Houwing S, Berezikov E, Ketting RF. 2008. Zili is required for germ cell differentiation and meiosis in zebrafish. EMBO $J$ 27: 2702-2711.

Huang HY, Houwing S, Kaaij LJ, Meppelink A, Redl S, Gauci S, Vos H, Draper BW, Moens CB, Burgering BM, et al. 2011. Tdrd1 acts as a molecular scaffold for Piwi proteins and piRNA targets in zebrafish. EMBO J 30: 3298-3308.

Ipsaro JJ, Haase AD, Knott SR, Joshua-Tor L, Hannon GJ. 2012. The structural biochemistry of Zucchini implicates it as a nuclease in piRNA biogenesis. Nature 491: 279-283.

Kamminga LM, Luteijn MJ, den Broeder MJ, Redl S, Kaaij LJ, Roovers EF, Ladurner P, Berezikov E, Ketting RF. 2010. Hen1 is required for oocyte development and piRNA stability in zebrafish. EMBO J 29: 3688-3700.

Kawaoka S, Izumi N, Katsuma S, Tomari Y. 2011. 3' end formation of PIWI-interacting RNAs in vitro. Mol Cell 43: 1015-1022.

Kawaoka S, Mitsutake H, Kiuchi T, Kobayashi M, Yoshikawa M, Suzuki Y, Sugano S, Shimada T, Kobayashi J, Tomari Y, et al. 2012. A role for transcription from a piRNA cluster in de novo piRNA production. RNA 18: 265-273.

Khurana JS, Wang J, Xu J, Koppetsch BS, Thomson TC, Nowosielska A, Li C, Zamore PD, Weng Z, Theurkauf WE. 2011. Adaptation to P element transposon invasion in Drosophila melanogaster. Cell 147: $1551-1563$.

Kidwell MG. 1983. Hybrid dysgenesis in Drosophila melanogaster: Factors affecting chromosomal contamination in the $P-M$ system. Genetics 104: 317-341. 
Klattenhoff C, Xi H, Li C, Lee S, Xu J, Khurana JS, Zhang F, Schultz N, Koppetsch BS, Nowosielska A, et al. 2009. The Drosophila HP1 homolog Rhino is required for transposon silencing and piRNA production by dual-strand clusters. Cell 138: 1137-1149.

Luteijn MJ, van Bergeijk P, Kaaij LJ, Almeida MV, Roovers EF, Berezikov E, Ketting RF. 2012. Extremely stable Piwi-induced gene silencing in Caenorhabditis elegans. EMBO J 31: 3422-3430.

Malone CD, Hannon GJ. 2009. Small RNAs as guardians of the genome. Cell 136: 656-668.

Muerdter F, Olovnikov I, Molaro A, Rozhkov NV, Czech B, Gordon A, Hannon GJ, Aravin AA. 2012. Production of artificial piRNAs in flies and mice. RNA 18: 42-52.

Nishimasu H, Ishizu H, Saito K, Fukuhara S, Kamatani MK, Bonnefond L, Matsumoto N, Nishizawa T, Nakanaga K, Aoki J, et al. 2012. Structure and function of Zucchini endoribonuclease in piRNA biogenesis. Nature 491: 284-287.

Pelisson A. 1981. The $I-R$ system of hybrid dysgenesis in Drosophila melanogaster: Are I factor insertions responsible for the mutator effect of the I-R interaction? Mol Gen Genet 183: 123-129.

Pelisson A, Song SU, Prud'homme N, Smith PA, Bucheton A, Corces VG. 1994. Gypsy transposition correlates with the production of a retroviral envelope-like protein under the tissuespecific control of the Drosophila flamenco gene. EMBO $J$ 13: 4401-4411.

Rozhkov NV, Aravin AA, Zelentsova ES, Schostak NG, Sachidanandam R, McCombie WR, Hannon GJ, Evgen'ev MB. 2010. Small
RNA-based silencing strategies for transposons in the process of invading Drosophila species. RNA 16: 1634-1645.

Sang HM, Pelisson A, Bucheton A, Finnegan DJ. 1984. Molecular lesions associated with white gene mutations induced by I-R hybrid dysgenesis in Drosophila melanogaster. EMBO J 3: 3079-3085.

Sarot E, Payen-Groschene G, Bucheton A, Pelisson A. 2004. Evidence for a piwi-dependent RNA silencing of the gypsy endogenous retrovirus by the Drosophila melanogaster flamenco gene. Genetics 166: $1313-1321$.

Shirayama M, Seth M, Lee HC, Gu W, Ishidate T, Conte D Jr, Mello CC. 2012. piRNAs initiate an epigenetic memory of nonself RNA in the C. elegans germline. Cell 150: 65-77.

Sienski G, Donertas D, Brennecke J. 2012. Transcriptional silencing of transposons by piwi and maelstrom and its impact on chromatin state and gene expression. Cell 151: 964-980.

Vagin VV, Sigova A, Li C, Seitz H, Gvozdev V, Zamore PD. 2006. A distinct small RNA pathway silences selfish genetic elements in the germline. Science 313: 320-324.

Voigt F, Reuter M, Kasaruho A, Schulz EC, Pillai RS, Barabas O. 2012. Crystal structure of the primary piRNA biogenesis factor Zucchini reveals similarity to the bacterial PLD endonuclease Nuc. RNA 18: 2128-2134.

Zhang F, Wang J, Xu J, Zhang Z, Koppetsch BS, Schultz N, Vreven T, Meignin C, Davis I, Zamore PD, et al. 2012. UAP56 couples piRNA clusters to the perinuclear transposon silencing machinery. Cell 151: 871-884. 

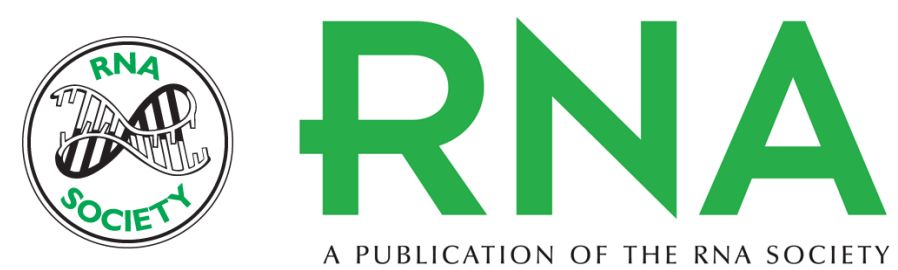

\section{piRNA dynamics in divergent zebrafish strains reveal long-lasting maternal influence on zygotic piRNA profiles}

Lucas J.T. Kaaij, Suzanne W. Hoogstrate, Eugene Berezikov, et al.

RNA 2013 19: 345-356 originally published online January 18, 2013

Access the most recent version at doi:10.1261/rna.036400.112

Supplemental
Material http://rnajournal.cshlp.org/content/suppl/2013/01/09/rna.036400.112.DC1

References This article cites 39 articles, 13 of which can be accessed free at:

http://rnajournal.cshlp.org/content/19/3/345.full.html\#ref-list-1

License

Email Alerting Receive free email alerts when new articles cite this article - sign up in the box at the Service top right corner of the article or click here. 